\title{
On the Computation of Some Grunsky Coefficients Relevant to the Bieberbach Conjecture
}

\author{
By George G. Ross
}

\begin{abstract}
The Bieberbach coefficients $a_{8}, a_{10}, a_{12}$ are parametrized in terms of the Grunsky coefficients. A digital computer is used to carry out the necessary integer calculations.
\end{abstract}

Introduction. The Bieberbach conjecture asserts that the $n$th coefficient of any univalent function

$$
f(z)=z+a_{2} z^{2}+a_{3} z^{3}+\cdots+a_{n} z^{n}+\cdots,
$$

defined in the unit circle, satisfies the inequality $\left|a_{n}\right| \leqq n$, with equality holding only for the Koebe function

$$
K(z)=z /(1-z)^{2}=z+2 z^{2}+3 z^{3}+\cdots
$$

and its one-parameter family of rotations $e^{-i \theta} K\left(e^{i \theta} z\right)$. The earliest results in this direction were Koebe's one-quarter theorem [6] and Bieberbach's proof of the sharp estimate $\left|a_{2}\right| \leqq 2$ [1]. Later Loewner [7] established the deeper inequality $\left|a_{3}\right| \leqq 3$ which has also been obtained by Schiffer, Schaeffer, and Spencer [9], [10], [11], [12] using variational methods. Garabedian and Schiffer [3] showed $\left|a_{4}\right| \leqq 4$ in 1960 and Pederson obtained the result $\left|a_{6}\right| \leqq 6$ in 1968 [8].

Of central importance in this theory, at least for the even coefficients, is the following theory of Grunsky [4]:

A necessary and sufficient condition for

$$
f(z)=z+a_{2} z^{2}+a_{3} z^{3}+\cdots
$$

to be univalent in the unit circle is that the sharp inequalities

$$
\operatorname{Re}\left\{\sum_{n, m=1}^{\infty} c_{n m} \lambda_{n} \lambda_{m}\right\} \leqq \sum_{n=1}^{\infty}\left|\lambda_{n}\right|^{2}
$$

hold for all choices of the complex parameters $\lambda_{n}$ where the $c_{n m}$ are the coefficients in the double power series

$$
\log \left[\frac{f(z)-f(w)}{z-w}\right]=\sum_{n, m=0}^{\infty} \frac{c_{n m}}{(n m)^{1 / 2}} z^{n} w^{m} .
$$

The above series converges in the bicylinder $|z| \leqq 1,|w| \leqq 1$ if and only if $f(z)$ is univalent.

The function $\left(f\left(z^{2}\right)\right)^{1 / 2}$ is easily seen to be univalent if and only if $f(z)$ is, and so we

Received August 7, 1970, revised March 17, 1971.

AMS 1970 subject classifications. Primary 30A34; Secondary 30-04.

Key words and phrases. Bieberbach conjecture, Koebe function, Grunsky coefficients, univalent functions, maximum principle, unitary matrix, Lagrange multipliers, overflow, fixed-point arithmetic. 
may generate coefficients $C_{n m}$ as polynomials in the $a_{i}$ from the expansion

$$
\log \left[\frac{\left(f\left(z^{2}\right)\right)^{1 / 2}-\left(f\left(w^{2}\right)\right)^{1 / 2}}{z-w}\right]=\sum_{n, m=0}^{\infty} C_{n m} z^{n} w^{m}
$$

and then impose the Grunsky inequalities on the $C_{n m}$ in order to get estimates on the Bieberbach coefficients $a_{i}$. A truncated set of these inequalities involving the $C_{n m}$ has been found sufficient to resolve the conjecture for $n=2,4,6$ and also to provide local confirmation for all even coefficients (Garabedian, Ross, Schiffer [2]). In that paper, it was shown that, for all univalent functions sufficiently "near" the Koebe function $K(z)$, the $2 n$th coefficient is not greater than $2 n$. Examination of the above work on the even coefficients suggests that the truncated set of Grunsky inequalities

$$
\operatorname{Re}\left\{\sum_{p, q=1}^{k}(2 p-1)^{1 / 2}(2 q-1)^{1 / 2} C_{2 p-1,2 q-1} \lambda_{2 p-1} \lambda_{2 q-1}\right\} \leqq \sum_{p=1}^{k}\left|\lambda_{2 p-1}\right|^{2}
$$

contains enough information to resolve the Bieberbach conjecture for $a_{2 k}$.

In this paper, we report on results obtained by introducing a computer to develop the relevant parametrizations of $a_{8}, a_{10}, a_{12}$ in terms of the $C_{2 p-1,2 q-1}$. These are the extensions of the formulas which, under the restrictions of the Grunsky inequalities, resolve the conjecture in the affirmative for $a_{2}, a_{4}, a_{6}$. It is believed that the imposition of the Grunsky conditions on the new expressions will settle the conjecture affirmatively for $a_{8}, a_{10}, a_{12}$. We already have mentioned that these parametrizations suffice to yield the result locally in a neighborhood of the Koebe function $K(z)$.

This work is a first step in a larger plan to develop algorithmic techniques which will help to resolve the Bieberbach conjecture through the use of a computer. The need for such techniques is evident when one notes the formidable amount of hand calculation involved in the highly nonlinear global proofs of $a_{4}$ [3] and $a_{6}$ [8]. It seems likely that future global results will rest on computer techniques and algorithms. These will simultaneously provide a check on the laborious hand calculations mentioned above which are too seldom checked by hand.

In Section II, we describe how the coefficients $C_{2 p-1,2 q-1}$ are generated as polynomials in the coefficients $a_{i}$ of the univalent $f(z)$.

In Section III, we develop the polynomial parametrizations of $a_{8}, a_{10}, a_{12}$ and also certain identities which exist between the elements $C_{2 p-1,2 q-1}$ of the parametrization.

Finally, in Section IV, we consider the relevant expressions for the Bieberbach coefficient $a_{8}$ and suggest how the maximum principle for several complex variables can be used to reduce the number of parameters involved. The role of the identities in the reduction is also discussed. The general technique for arriving at a parametrization which works globally for $a_{2 m}$ is illustrated.

Note. The appendices referred to in the text appear in the microfiche section at the end of this issue of Mathematics of Computation.

II. This section describes the development of the Grunsky coefficients $C_{i j}$ $(i, j$ odd) in the double power series

$$
\log \left[\frac{\left(f\left(z^{2}\right)\right)^{1 / 2}-\left(f\left(w^{2}\right)\right)^{1 / 2}}{z-w}\right]=\sum_{i, j=0}^{\infty} C_{i j} z^{i} w^{i} .
$$

The $C_{i j}$ are polynomials in the $a_{n}$ coefficients of the univalent function 


$$
f(z)=z+a_{2} z^{2}+a_{3} z^{3}+\cdots .
$$

To carry out the development we write

$$
\left(f\left(z^{2}\right)\right)^{1 / 2}=z\left(1+a_{2} z^{2}+a_{3} z^{4}+\cdots+a_{n} z^{2(n-1)}+\cdots\right)^{1 / 2}
$$

and apply the multinomial theorem to the term

$$
x(z)=a_{2} z^{2}+a_{3} z^{4}+\cdots
$$

in the expansion

$$
(1+x(z))^{1 / 2}=1+\frac{1}{2} x(z)-\frac{1}{8} x^{2}(z)+\cdots .
$$

This yields the series expansion for $\left(f\left(z^{2}\right)\right)^{1 / 2}$ in the variable $z$. If we subtract the series for $\left(f\left(w^{2}\right)\right)^{1 / 2}$ from the series for $\left(f\left(z^{2}\right)\right)^{1 / 2}$ and divide by the factor $z-w$, we have

$$
\begin{aligned}
\frac{\left(f\left(z^{2}\right)\right)^{1 / 2}-\left(f\left(w^{2}\right)\right)^{1 / 2}}{z-w} & =1+\frac{1}{2} a_{2}\left(z^{2}+z w+w^{2}\right) \\
& +\left(a_{3}-\frac{1}{8} a_{2}^{2}\right)\left(z^{4}+z^{3} w+z^{2} w^{2}+z w^{3}+w^{4}\right)+\cdots \\
& +P_{n}\left(a_{i}\right)\left(z^{2 n}+z^{2 n-1} w+\cdots+w^{2 n}\right)+\cdots,
\end{aligned}
$$

where the $P_{n}$ are the polynomials in the $a_{i}$ determined by the square root expansion and the multinomial theorem. The final formula for the $C_{i j}$ is attained by identifying the expansion above with $1+Y(z, w)$ and again applying the multinomial theorem to the quantity $Y$ in the series

$$
\log [1+Y(z, w)]=Y(z, w)-\frac{Y^{2}(z, w)}{2}+\frac{Y^{3}(z, w)}{3}+\cdots .
$$

The final expression is

$$
C_{i j}=\sum C_{i j}\left(\alpha_{1}, \alpha_{2}, \cdots, \alpha_{l}\right) a_{2}^{\alpha_{1}} a_{3}^{\alpha_{2}} \cdots a_{l+1}^{\alpha_{l}}
$$

over all nonnegative $\alpha_{i}$ such that

$$
\alpha_{1}+2 \alpha_{2}+\cdots+l \alpha_{l}=l=i+j,
$$

where

$$
\begin{aligned}
C_{i j}\left(\alpha_{1}, \alpha_{2}, \cdots, \alpha_{l}\right) \\
\quad=\sum_{k=0}^{[i / 2\}} \frac{(-1)^{(A-S+1)}}{2(i / 2-k+A-S)}\left(\begin{array}{c}
i / 2-k+A-S \\
s_{1}, s_{2}, \cdots, s_{l}, \alpha_{1}-s_{1}, \cdots, \alpha_{l}-s_{l}
\end{array}\right),
\end{aligned}
$$

and the inner summation is performed over all $s_{n}$ such that

$$
0 \leqq s_{n} \leqq \alpha_{n} \text { and } s_{1}+2 s_{2}+\cdots+l s_{l}=k \text {. }
$$

In the above,

$$
A=\alpha_{1}+\alpha_{2}+\cdots+\alpha_{l}, \quad S=s_{1}+s_{2}+\cdots+s_{l}
$$

and [ ] denotes integer part.

These formulas agree with a more general set of formulas for Grunsky coefficients worked out by Hummel [5].

A Fortran program was written to implement the above expressions on an IBM $360 / 50$ computer. All calculations were done in fixed-point arithmetic to insure 
precise results. The basic arithmetic operations involved are the addition and multiplication of ratios of integers. Denominators other than one are generated by the factorial coefficients appearing in the expansions of $(1+x)^{1 / 2}$ and $\log (1+Y)$. The program quickly produced overflow in the IBM 32-bit fixed-point calculation, and a special routine performing Euclid's algorithm was included to reduce $a / b+c / d$ to lowest terms. Finally, the program was run on the CDC 6600 where 48-bit fixed-point arithmetic is simulated. On this machine, the program successfully calculated all $C_{i j}(i, j$ odd), $i, j \leqq 11$. These results appear in Appendix A.

The results can be extended for higher $i, j$, either by using the present program on a machine with larger word size or by modifying it so that the arithmetic operations are carried out by a multi-precision subroutine.

III. In this section, we convert the expression obtained in the last section into formulas for the $a_{n}$ as polynomials in the $C_{2 p-1,2 q-1}$. We have a double array of coefficients $C_{2 p-1,2 q-1}$ which depend on the single sequence of variables $a_{2}, a_{3}, \cdots, a_{n}$ with $n \leqq p+q$. Thus, identities arise between the elements $C_{2 p-1,2 q-1}$. The first such identity occurs between the elements $C_{11}, C_{13}, C_{15}, C_{33}$ :

$$
C_{15}=-\frac{1}{3} C_{11}^{3}+\frac{1}{3} C_{33}+C_{11} C_{13} .
$$

Appendix A shows clearly the existence of other identities. In fact, the $k(k+1) / 2$ elements of the symmetric matrix $C_{2 p-1,2 q-1}(p, q \leqq k)$ depend on the $2 k-1$ coefficients $a_{2}, a_{3}, \cdots, a_{k}, \cdots, a_{2 k}$. Hence, there are

$$
\frac{k(k+1)}{2}-2 k+1=\frac{k^{2}}{2}-\frac{3}{2} k+1
$$

identities between these $C_{2 p-1,2 q-1}$.

The formula manipulation involved in generating the $a_{2 k}$ as polynomials in the $C_{2 p-1,2 q-1}$ and in generating the attendant identities was carried out by means of an assembly language program written for the IBM $360 / 50$. The program first sets $a_{2}=2 C_{11}$ and then proceeds recursively for $p+q \geqq 3$ through expressions for the $C_{2 p-1,2 q-1}$ given in Appendix A. If $p=q$, the expression for $a_{2 p}$ is computed by replacing all $a_{n}$ with $n<2 p$ by previously computed polynomials in the $C_{2 p-1,2 q-1}$. If $p=q-1$, the expression for $a_{2 p-1}$ is computed in the same way. If $p=q-2$, an identity is generated by replacing all $a_{n}$ in the expression by previously computed polynomials in the $C_{2 p-1,2 q-1}$. The validity of the algorithm is established by observing:

(1) $C_{2 p-1,2 p-1}$ is linear in $a_{2 p}$,

(2) $C_{2 p-3,2 p-1}$ is linear in $a_{2 p-1}$,

(3) $C_{2 p-1,2 q-1}$ depends only on $a_{2}, a_{3}, \cdots, a_{2 q-2}$ if $p \leqq q-2$.

Throughout the calculation, numerical coefficients were carried as the ratio of fixed-point integers to avoid loss of precision. Overflow problems on the IBM 360/50 demanded the same delicacy of arithmetic manipulation discussed in the previous section. Results of the computation appear in Appendix B.

The author acknowledges the major contribution of Mr. Aaron W. Weg in his assistance in the preparation and debugging of this program.

IV. The polynomial expressions for $a_{2 n}$, given in Appendix B, must be subjected to the Grunsky conditions 


$$
\operatorname{Re}\left\{\sum_{p, q=1}^{n}(2 p-1)^{1 / 2}(2 q-1)^{1 / 2} C_{2 p-1,2 q-1} \lambda_{2 p-1} \lambda_{2 q-1}\right\} \leqq \sum_{p=1}^{n}\left|\lambda_{2 p-1}\right|^{2} .
$$

A result of Schur's [13] enables us to rewrite the above conditions in matrix notation as

$$
\bar{C}=U D U^{T},
$$

where $\bar{C}$ is an $n$th order square symmetric matrix with elements

$$
(2 p-1)^{1 / 2}(2 q-1)^{1 / 2} C_{2 p-1,2 q-1} \quad(p, q \leqq n),
$$

$U$ is a unitary $n$th order matrix, and $D$ is a diagonal matrix whose entries have absolute value less than or equal to one. In previous work [2], the maximum principle for several complex variables was invoked to show that for the maximum modulus of $a_{2 n}$, the diagonal elements of $D$ actually assume the value one. Thus, $C$ becomes simultaneously unitary and symmetric. Maximization over all such unitary matrices has yielded the Bieberbach conjecture for $a_{4}$ and $a_{6}$.

An application of the new formulas generated is made to the study of the eighth Bieberbach coefficient. The parametrization for $a_{8}$ from Appendix B is

$$
\begin{aligned}
a_{8}= & \frac{2}{7} \bar{C}_{77}+\frac{16}{3 \sqrt{ } 15} \bar{C}_{33} \bar{C}_{35}+\frac{12}{5 \sqrt{ } 3} \bar{C}_{13} \bar{C}_{55}+\frac{46}{9} \bar{C}_{13}^{2} \bar{C}_{33}+\frac{8}{\sqrt{ } 35} \bar{C}_{11} \bar{C}_{57} \\
& +\frac{34}{9} \bar{C}_{11} \bar{C}_{33}^{2}+\frac{64}{3 \sqrt{ } 5} \bar{C}_{11} \bar{C}_{13} \bar{C}_{35}+\frac{76}{3 \sqrt{ } 3} \bar{C}_{11} \bar{C}_{13}^{3}+\frac{18}{5} \bar{C}_{11}^{2} \bar{C}_{55} \\
& +\frac{172}{3 \sqrt{ } 3} \bar{C}_{11}^{2} \bar{C}_{13} \bar{C}_{33}+\frac{80}{3 \sqrt{ } 15} \bar{C}_{11}^{3} \bar{C}_{35}+\frac{410}{9} \bar{C}_{11}^{3} \bar{C}_{13}^{2}+\frac{82}{9} \bar{C}_{11}^{4} \bar{C}_{33} \\
& +\frac{184}{15 \sqrt{ } 3} \bar{C}_{11}^{5} \bar{C}_{13}-\frac{2764}{315}-\bar{C}_{11}^{7} .
\end{aligned}
$$

Because the ten elements $C_{2 p-1,2 q-1}, p, q \leqq 4$, depend only on the seven coefficients $a_{2}, a_{3}, a_{4}, a_{5}, a_{6}, a_{7}, a_{8}$, there are three identities which hold between these elements. They are (from Appendix B)

Identity 1: $\quad-\frac{\bar{C}_{15}}{\sqrt{ } 5}+\frac{\bar{C}_{33}}{3}+\frac{\bar{C}_{11} \bar{C}_{13}}{\sqrt{ } 3}-\frac{\bar{C}_{11}^{3}}{3}=0$,

Identity 2: $\quad-\frac{\bar{C}_{17}}{\sqrt{ } 7}+\frac{\bar{C}_{35}}{\sqrt{ } 15}+\frac{\bar{C}_{13}^{2}}{3}+\frac{\bar{C}_{11} \bar{C}_{33}}{3}-\frac{\bar{C}_{11}^{4}}{3}=0$,

Identity 3: $\quad-\frac{\bar{C}_{37}}{\sqrt{ } 21}+\frac{\bar{C}_{55}}{5}+\frac{\bar{C}_{13} \bar{C}_{33}}{3 \sqrt{ } 3}-\frac{\bar{C}_{11} \bar{C}_{13}^{2}}{3}+\frac{2 \bar{C}_{11}^{3} \bar{C}_{13}}{2 \sqrt{ } 3}-\frac{\bar{C}_{11}^{5}}{5}=0$.

If we assume the $\bar{C}_{2 p-1,2 q-1}$ are independent complex variables subject only to the Grunsky inequalities in the formula for $a_{8}$, we can apply the maximum principle for several complex variables to obtain a significant reduction in the twenty parameters required to represent the complex symmetric matrix

$$
\left(\bar{C}_{2 p-1}, \bar{C}_{2 q-1}\right), \quad p, q \leqq 4 .
$$

We have mentioned above that the greatest modulus of $a_{2 k}$ must be assumed when the above matrix satisfies unitary constraints and so requires only ten parameters to represent it. But our assumption of independent variation of the $C_{2 p-1,2 q-1}$ precludes the possibility of imposing the above identities on the elements of the unitary matrix 


$$
\left(\begin{array}{llll}
\bar{C}_{11} & \bar{C}_{13} & \bar{C}_{15} & \bar{C}_{17} \\
\bar{C}_{13} & \bar{C}_{33} & \bar{C}_{35} & \bar{C}_{37} \\
\bar{C}_{15} & \bar{C}_{35} & \bar{C}_{55} & \bar{C}_{57} \\
\bar{C}_{17} & \bar{C}_{37} & \bar{C}_{57} & \bar{C}_{77}
\end{array}\right) .
$$

To compensate for this situation, we exploit the flexibility in choice of the formula for $a_{8}$ which the identities provide. We add multiples of the three identities to $a_{8}$ in the form of Lagrange constraints. We form

$$
\begin{aligned}
& a_{8}+\left(\lambda_{1} \bar{C}_{11}^{4}+\lambda_{2} \bar{C}_{11}^{2} \bar{C}_{13}+\lambda_{3} \bar{C}_{11} \bar{C}_{15}+\lambda_{4} \bar{C}_{11} \bar{C}_{33}+\lambda_{5} \bar{C}_{17}+\lambda_{6} \bar{C}_{35}\right) \text { Id } 1 \\
& \quad+\left(\lambda_{7} \bar{C}_{11}^{3}+\lambda_{8} \bar{C}_{11} \bar{C}_{13}+\lambda_{9} \bar{C}_{33}+\lambda_{10} \bar{C}_{15}\right) \text { Id } 2+\left(\lambda_{11} \bar{C}_{11}^{2}+\lambda_{12} \bar{C}_{13}\right) \text { Id } 3 \\
& \quad+\lambda_{13} \bar{C}_{11}(\text { Id } 1)^{2},
\end{aligned}
$$

where

Id 1 is the left-hand side of Identity 1 ,

Id 2 is the left-hand side of Identity 2 ,

Id 3 is the left-hand side of Identity 3.

In our selection of parameters, we have preserved the homogeneity of $a_{8}$ in the sense that, if $\bar{C}_{n m}$ is replaced by $\bar{C}_{n m} e^{i \theta n} c^{i \theta m}$, then a factor $e^{14 i \theta}$ is brought out of the new expression for $a_{8}$. This homogeneity has the desirable feature that the rotation above not only leaves invariant the class of matrices $\bar{C}_{2 p-1,2 q-1}$ obeying Grunsky's inequalities, but also leaves the value of $\left|a_{8}\right|$ fixed.

We now seek to choose the parameters $\lambda_{i}$ so that, when the unitary symmetric matrix is represented in the form $\bar{C}_{2 p-1,2 q-1}=e^{i A}(p, q \leqq 4, A$ a real symmetric $4 \times 4$ matrix), the resulting expression for $\operatorname{Re}\left\{a_{8}\right\}$ will have a local maximum with respect to the 10 elements of $A$ at $A \equiv 0$. The situation $A \equiv 0$ corresponds to $\left\{\bar{C}_{2 p-1,2 q-1}\right\}$ equal to the identity matrix and to $F(z)$ equal to the Koebe function $K(z)$. Clearly, if our formula for $a_{8}$ is to yield the Bieberbach conjecture, it must have a local maximum at the Koebe point. Experience has indicated that those formulas which confirm the conjecture locally at $K(z)$ can be extended to global confirmation of the conjecture (cf. (2)).

To carry out this choice of $\lambda_{i}$, we write out the exponential expansion up to quadratic terms in the elements of $A=\left(a_{i j}\right)$ :

$$
e^{i A}=I+i A-\frac{1}{2}\left(A^{2}\right) .
$$

We then have:

$$
\begin{aligned}
& \bar{C}_{11}=1+i a_{11}-\frac{1}{2}\left(a_{11}^{2}+a_{12}^{2}+a_{13}^{2}+a_{14}^{2}\right), \\
& \bar{C}_{13}=0+i a_{12}-\frac{1}{2}\left(a_{11} a_{12}+a_{12} a_{22}+a_{13} a_{23}+a_{14} a_{24}\right), \\
& \bar{C}_{15}=0+i a_{13}-\frac{1}{2}\left(a_{11} a_{13}+a_{12} a_{23}+a_{13} a_{33}+a_{14} a_{34}\right), \\
& \bar{C}_{17}=0+i a_{14}-\frac{1}{2}\left(a_{11} a_{14}+a_{12} a_{24}+a_{13} a_{34}+a_{14} a_{44}\right), \\
& \bar{C}_{33}=1+i a_{22}-\frac{1}{2}\left(a_{12}^{2}+a_{22}^{2}+a_{23}^{2}+a_{24}^{2}\right), \\
& \bar{C}_{35}=0+i a_{23}-\frac{1}{2}\left(a_{12} a_{13}+a_{22} a_{23}+a_{23} a_{33}+a_{24} a_{34}\right),
\end{aligned}
$$




$$
\begin{aligned}
& \bar{C}_{37}=0+i a_{24}-\frac{1}{2}\left(a_{12} a_{14}+a_{22} a_{24}+a_{23} a_{34}+a_{24} a_{44}\right), \\
& \bar{C}_{55}=1+i a_{33}-\frac{1}{2}\left(a_{13}^{2}+a_{23}^{2}+a_{24}^{2}+a_{34}^{2}\right), \\
& \bar{C}_{57}=0+i a_{34}-\frac{1}{2}\left(a_{13} a_{14}+a_{23} a_{24}+a_{33} a_{34}+a_{34} a_{44}\right), \\
& \bar{C}_{77}=1+i a_{44}-\frac{1}{2}\left(a_{14}^{2}+a_{24}^{2}+a_{34}^{2}+a_{44}^{2}\right) .
\end{aligned}
$$

We next calculate the constant, linear, and quadratic parts of $\operatorname{Re} a_{8}$ and of each expression to be added to it. There is one such expression for each $\lambda_{i}$ in expression (1). Special routines were written to carry out the arithmetic involved in the tedious computation of the local behavior of $a_{8}$ and the identities. The routines did addition, subtraction, and multiplication of quantities of the form

$$
\begin{aligned}
& \left.C_{0}+C_{1} i \text { (linear form in the } a_{i j}, i, j=1,4\right) \\
& +C_{2} \text { (quadratic form in the } a_{i j}, i, j=1,4 \text { ), }
\end{aligned}
$$

producing a result of the same form and neglecting the resulting higher order terms. It remains to choose the $\lambda_{i}$ so that $\operatorname{Re}\left\{a_{8}\right\}$ has a negative definite second variation at $A \equiv 0$. We observe that we lose no generality if we choose $\lambda_{i}$ real, since a complex part of $\lambda_{i}$ would influence only the complex part of $a_{8}$. Note the first variation of $\operatorname{Re}\left\{a_{8}\right\}$ at $A \equiv 0$ is 0 since all coefficients are real.

We set

$A_{0}=$ matrix corresponding to quadratic form of $\operatorname{Re} a_{8}$,

$A_{1}=$ matrix corresponding to quadratic form of $\bar{C}_{11}^{4}$ Id 1 ,

$A_{2}=$ matrix corresponding to quadratic form of $\bar{C}_{11}^{2} \bar{C}_{13}$ Id 1 ,

$A_{3}=$ matrix corresponding to quadratic form of $\bar{C}_{11} \bar{C}_{15} \operatorname{Id~} 1$,

$A_{4}=$ matrix corresponding to quadratic form of $\bar{C}_{11} \bar{C}_{33} \mathrm{Id} 1$,

$A_{5}=$ matrix corresponding to quadratic form of $\bar{C}_{17} \operatorname{Id} 1$,

$A_{6}=$ matrix corresponding to quadratic form of $\bar{C}_{35}$ Id 1 ,

$A_{7}=$ matrix corresponding to quadratic form of $\bar{C}_{11}^{3} \operatorname{Id~} 2$,

$A_{8}=$ matrix corresponding to quadratic form of $\bar{C}_{11} \bar{C}_{13} \operatorname{Id} 2$,

$A_{9}=$ matrix corresponding to quadratic form of $\bar{C}_{33}$ Id 2 ,

$A_{10}=$ matrix corresponding to quadratic form of $\bar{C}_{15} \mathrm{Id} 2$,

$A_{11}=$ matrix corresponding to quadratic form of $\bar{C}_{11}^{2}$ Id 3 ,

$A_{12}=$ matrix corresponding to quadratic form of $\bar{C}_{13}$ Id 3 ,

$A_{13}=$ matrix corresponding to quadratic form of $\bar{C}_{11}$ (Id 1$)^{2}$.

We form

$$
A_{0}+\sum_{i=1}^{13} \lambda_{i} A_{i}
$$

for a choice of $\lambda_{i}$ and then use a standard eigenvalue code to find the maximum eigenvalue of the above. We introduced various minimization schemes to minimize 
this maximum eigenvalue with respect to the parameters $\lambda_{i}$. We succeeded in finding a set of $\lambda_{i}$ for which the largest eigenvalue was negative, thus guaranteeing the negative definiteness of the second variation of the formula for $\operatorname{Re} a_{8}$ and having therefore the desired local behavior at the Koebe point. We found that the choice of parameters

$$
\begin{aligned}
& \lambda_{1}=-19.235992432 \quad \lambda_{2}=-90.875000000 \quad \lambda_{3}=-1.187500000 \\
& \lambda_{4}=-4.555971146 \quad \lambda_{5}=-27.875000000 \quad \lambda_{6}=12.437500000 \\
& \lambda_{7}=-12.254911423 \quad \lambda_{8}=-20.169921875 \quad \lambda_{9}=0.000000000 \\
& \lambda_{10}=-0.937500000 \quad \lambda_{11}=-9.843718529 \quad \lambda_{12}=-6.562591553 \\
& \lambda_{13}=1837.312500000
\end{aligned}
$$

produced $A_{0}+\sum_{i=1}^{13} \lambda_{i} A_{i}$ shown below. The maximum eigenvalue of this matrix is less than -0.002 .

$$
\left[\begin{array}{rrrrrr}
-1933.339111 & 987.395752 & -843.616699 & -22.043350 & 609.912354 \\
987.395752 & -624.305420 & 453.139893 & 3.696342 & -354.901123 \\
-843.616699 & 453.139893 & -384.766602 & -6.748270 & 273.225098 \\
-22.043350 & 3.696342 & -6.748270 & -16.103760 & 4.645832 \\
609.912354 & -354.901123 & 273.225098 & 4.645832 & -208.730331 \\
0.637105 & -8.416772 & -2.630764 & 0.0 & -4.035986 \\
-2.154895 & -1.880836 & 0.0 & -5.532904 & -0.538724 \\
-1.625007 & -0.030312 & -2.660021 & 0.0 & 0.0 \\
-0.676123 & 0.0 & -1.157980 & -2.660021 & 0.0 \\
0.0 & 0.0 & 0.0 & -1.157980 & 0.0 \\
0.0 & & & & & \\
& 0.637105 & -2.154895 & -1.625007 & -0.676123 & 0.0 \\
& -8.416772 & -1.880836 & -0.030312 & 0.0 & 0.0 \\
& -2.630764 & 0.0 & -2.660021 & -1.157980 & 0.0 \\
& 0.0 & -5.532904 & 0.0 & -2.660021 & -1.157980 \\
& -4.035986 & -0.538724 & 0.0 & 0.0 & 0.0 \\
& -3.138024 & -0.338062 & -1.274540 & -0.538724 & 0.0 \\
& -0.338062 & -2.468379 & 0.0 & -1.274540 & -0.538724 \\
& -1.274540 & 0.0 & -0.812503 & -0.338062 & 0.0 \\
& -0.538724 & -1.274540 & -0.338062 & -0.955359 & -0.338062 \\
0.0 & -0.538724 & 0.0 & -0.338062 & -0.142857
\end{array}\right] .
$$

The final expression for $a_{8}$ corresponding to the choice of parameters above is

$$
\begin{aligned}
a_{8}= & 207.8369 \bar{C}_{11}^{7}-675.0666 \bar{C}_{11}^{5} \bar{C}_{13}-408.1586 \bar{C}_{11}^{4} \bar{C}_{33}+557.0915 \bar{C}_{11}^{4} \bar{C}_{15} \\
& +13.9236 \bar{C}_{11}^{3} \bar{C}_{17}-0.4247 \bar{C}_{11}^{3} \bar{C}_{35}+600.2736 \bar{C}_{11}^{3} \bar{C}_{13}^{2}+1.6313 \bar{C}_{11}^{2} \bar{C}_{55} \\
& +698.7446 \bar{C}_{11} \bar{C}_{13}^{3}-908.8291 \bar{C}_{11}^{2} \bar{C}_{13} \bar{C}_{15}+206.4049 \bar{C}_{11} \bar{C}_{33}^{2}+3.04255 \bar{C}_{11} \bar{C}_{57} \\
& +11.5142 \bar{C}_{11} \bar{C}_{13} \bar{C}_{35}+10.0914 \bar{C}_{11} \bar{C}_{13}^{3}+367.9934 \bar{C}_{11} \bar{C}_{15}^{2} \\
& -546.4514 \bar{C}_{11} \bar{C}_{33} \bar{C}_{15}-8.4712 \bar{C}_{11} \bar{C}_{13} \bar{C}_{17}+2.1481 \bar{C}_{11}^{2} \bar{C}_{37}+5.5229 \bar{C}_{33} \bar{C}_{35} \\
& +0.0731 \bar{C}_{13} \bar{C}_{55}+3.8481 \bar{C}_{13}^{2} \bar{C}_{33}+12.8204 \bar{C}_{17} \bar{C}_{15}-0.3125 \bar{C}_{13}^{2} \bar{C}_{15} \\
& -5.8043 \bar{C}_{15} \bar{C}_{35}-9.2917 \bar{C}_{17} \bar{C}_{33}+1.4321 \bar{C}_{13} \bar{C}_{37}+0.2857 \bar{C}_{77} .
\end{aligned}
$$


All coefficients have been truncated at four decimal places.

Preliminary numerical testing of the expression with the $\bar{C}_{2 p-1,2 q-1}$, bound by unitary constraints, indicates the inequality

$$
\operatorname{Re} a_{8} \leqq 8
$$

holds for all parameter choices. Rigorous confirmation of this, of course, would establish the conjecture for the 8th coefficient.

Computer Sciences Department

City College of New York

New York, New York 10031

1. L. Bieberbach, "Ueber die Koeffizienten derjenigen Potenzreihen, welche eine schlichte Abbildung des Einheitkreises vermitteln," S.-B. Preuss. Akad. Wiss., v. 138, 1916. pp. $940-955$.

2. P. R. Garabedian, G. Ross \& M. Schiffer, "On the Bieberbach conjecture for even n," J. Math. Mech., v. 14, 1965, pp. 975-989. MR 32 \#207.

3. P. R. GARABEDIAN \& M. SCHIFFER, "A proof of the Bieberbach conjecture for the fourth coefficient," J. Rational Mech. Anal., v. 4, 1955, pp. 427-465. MR 17, 24.

4. H. GRUNSKY, "Koeffizientenbedingungen für schlicht abbildende meromorphe Funktionen," Math. Z., v. 45, 1939, pp. 29-61.

5. J. A. Hummel, "The Grunsky coefficients of schlicht functions," Proc. Amer. Math. Soc., v. 15, 1964, pp. 142-150. MR 28 \#1287.

6. P. KoEBE, "Ueber die Uniformisierung der algebraischen Kurven. II," Math. Ann., v. 69,1910 , pp. $1-81$.

7. K. LOEWNER, "Untersuchungen ueber schlichte konforme Abbildungen des Einheitskreises. I," Math. Ann., v. 89, 1923, pp. 103-121.

8. R. N. PEDERSON, "A proof of the Bieberbach conjecture for the sixth coefficient," Arch. Rational Mech. Anal., v. 31, 1968/69, pp. 331-351. MR 39 \#31.

9. M. SCHIFFER, "A method of variation within the family of simple functions," Proc. London Math. Soc., v. 44, 1938, pp. 432-449.

10. M. SCHIFFER, "Variation of the Green function and theory of the p-valued functions," Amer. J. Math., v. 65, 1943, pp. 341-360. MR 4, 215.

11. A. C. SCHAEFFER \& D. C. SPENCER, "The coefficients of schlicht functions," Duke Math. J., v. 10, 1943, pp. 611-635. MR 5, 175 .

12. A. C. SCHAEFFER \& D. C. SPENCER, Coefficient Regions for schlicht Functions, Amer. Math. Soc. Colloq. Publ., vol. 35, Amer. Math. Soc., Providence, R.I., 1950. MR 12, 326.

13. I. ScHUR, "Ein Satz ueber quadratische Formen mit komplexen Koeffizienten," Amer. J. Math., v. 67, 1945, pp. 472-480. 